\title{
Residual Gas X-ray Beam Position Monitor Development for PETRA III
}

\author{
P. Ilinski, U. Hahn, H. Schulte-Schrepping, M. Degenhardt \\ HASYLAB, DESY, Notkestr. 85, 22607 Hamburg, Germany
}

\begin{abstract}
The development effort is driven by the need for a new type of x-ray beam position monitor (XBPM), which will detect the centre of gravity of the undulator beam. XBPMs based on the ionization of a residual gas are considered being the candidate for this future "white" undulator beam XBPMs. A number of residual gas XBPM prototypes for the PETRA III storage ring were developed and tested. Tests were performed at DESY and the ESRF, resolution of beam position up to $5 \mu \mathrm{m}$ is reported. The further development of the RGXBPMs will be focused on improvements of resolution, readout speed and reliability.
\end{abstract}

Keywords: synchrotron radiation, undulator radiation, x-ray beam position monitor, residual gas

PACS: 07.85 .Qe

\section{INTRODUCTION}

Blade type x-ray beam position monitors (XBPM) are currently employed at the third generation synchrotron facilities as "white" undulator beam XBPMs $[1,2]$. They provide a micron accuracy resolution and are capable to withstand the high power of the undulator radiation. Nevertheless since the information of the beam position is obtained from the halo of the undulator radiation, the signal depends on the undulator gap and is affected by stray radiation from bending magnets and focusing optics. These can be illuminated in case of XBPM, which will detect the centre of gravity of the beam. XBPMs based on the ionization of a residual gas can be considered as a candidate for this future "white" beam XBPMs. Residual gas XBPM (RGXBPM) have no elements which are hit by the beam, may have wide dynamic range and will directly detect the centre of gravity of the undulator radiation.

\section{RESIDUAL GAS X-RAY BEAM POSITION MONITOR}

Residual gas beam profile monitors were first developed to provide beam profile measurements at charged particles accelerators [3,4] and currently are in use at High Energy Physics facilities [5]. The profile monitor consists of an ion chamber operated at a residual gas pressure in which ions or electrons are drifted in a parallel electrical field towards the micro channel plate (MCP), which amplifies the signal and produces an image of the beam profile on a phosphor screen, Fig. 1a.

\section{Resolution}

The resolution of the residual gas beam profile monitors, which are in use at the particle accelerators is in the order of few hundreds of microns. Resolution of RGXBPM has to be substantially improved in order to comply with the requirements at the PETRA III storage ring. Factors which define the RGXBPM resolution are: quality of the electrical field, initial kinetic energy of ions or electrons, resolution of detection system, and data processing. The electrical field has to be uniform in order to provide aberration free beam profile. Broadening of the beam profile occurs due to electrical field non uniformity and presence of the transverse component of the electrical field. This broadening should not exceed the broadening of the beam profile which is caused by the initial transverse kinetic energy of ions or electrons [6]. The resolution of detection system is defined by the MCP, the phosphor screen, optical coupling and signal to background ratio. A proper data processing allows for sub-pixel resolution, where pixel characterizes the resolution of the detection system.

CP879, Synchrotron Radiation Instrumentation: Ninth International Conference, edited by Jae-Young Choi and Seungyu Rah

(C) 2007 American Institute of Physics 978-0-7354-0373-4/07/\$23.00 


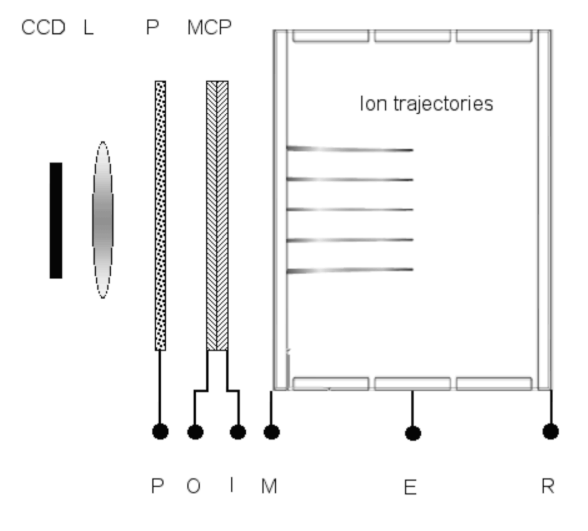

(a)

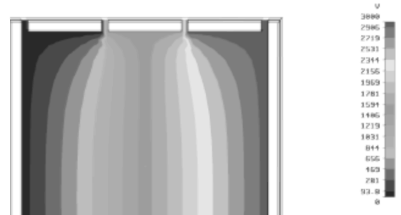

(b)

FIGURE 1. RGXBPM general layout: R- repeller electrode, $\mathrm{E}$ - guide electrode, $\mathrm{M}$ - mesh window electrode, I - MCP input, $\mathrm{O}$ - MCP output, $\mathrm{P}$ - phosphor screen, L - objective lens, CCD camera (a). RGXBPM potential distribution (b).

\section{Signal Level}

The signal level of the RGXBPM first of all is defined by the total x-ray crossection of the residual gas, which depends on pressure and gas composition. Typical pressure at the beamline can be better then $10^{-9}$ mbar, gas composition may vary, and be mostly hydrogen or include nitrogen, water vapor etc. In a typical high pressure ion chamber a primary photoelectron will produce an avalanche of secondary electrons, so the number of created electron ion pairs will be proportional to deposited energy divided by average energy needed for one electron ion pair creation ( $35 \mathrm{eV}$ for nitrogen). In the RGXBPM, since residual gas pressure is low, the primary photoelectrons may not produce secondary electrons. Two scenarios are presented at Fig. 2a for nitrogen and hydrogen at pressure of $10^{-6} \mathrm{mbar}$, and for detector length of $1 \mathrm{~cm}$. First, when only primary photoelectrons are detected: (dots) nitrogen, (dash-dot-dot) - hydrogen, second, when all secondary electrons where produced and detected: (dashed) nitrogen, (dash-dot) - hydrogen.

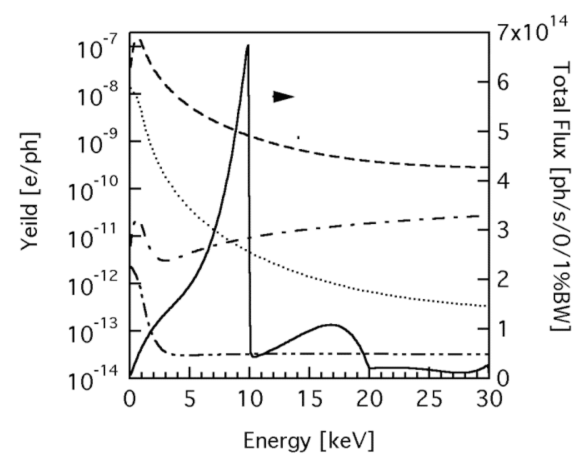

(a)

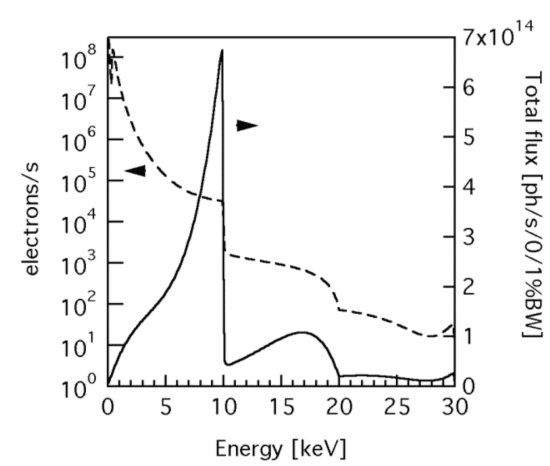

(b)

FIGURE 2. Total flux of PETRA III undulator, $2.9 \mathrm{~cm}$ period length, total length of $2 \mathrm{~m}, \mathrm{~K}=0.6$ (a, b). Electrons/ions yield at gas pressure of $10^{-6} \mathrm{mbar}$, and collection length of $1 \mathrm{~cm}$; all secondary electrons are produced and collected: nitrogen (dashed), hydrogen (dashed-dot), only primary photoelectrons are collected: nitrogen (dots), hydrogen (dash-dot-dot) (a). Photoelectron current, when only primary photoelectrons are collected (nitrogen gas) (b).

A photoelectron current, when only photoelectrons are collected, is presented at Fig. 2b for PETRA III undulator of $2.9 \mathrm{~cm}$ period length, total length of $2 \mathrm{~m}$, and for $\mathrm{K}$ value of 0.6 . As can be seen most of the photoelectrons are generated at low energies, even if the undulator radiation intensity is extremely low at such energies. Undulator radiation, which is "red shifted" from fundamental harmonic undulator radiation, is radiated off axis. The undulator flux distribution of $1 \mathrm{keV}$ photons at $20 \mathrm{~m}$ for above undulator parameters is presented at Fig. 3b. In this case a complete profile picture may be complicated. In case when the signal is proportional to the deposited energy, the beam profile will be formed by the power distribution of the undulator radiation, shown at Fig $3 \mathrm{a}$. 


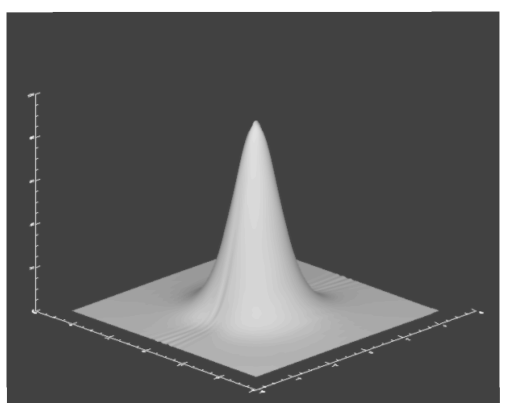

(a)

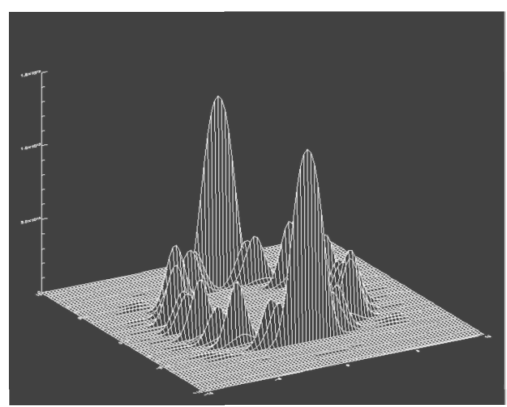

(b)

FIGURE 3. PETRA III undulator, $2.9 \mathrm{~cm}$ period length, total length of $2 \mathrm{~m}, \mathrm{~K}=0.6$. Distribution at $20 \mathrm{~m}$ of undulator radiation power density $\left(10 \times 10 \mathrm{~mm}^{2}\right)(\mathrm{a})$, and flux density spatial distribution for $1 \mathrm{keV}$ energy photons $\left(20 \times 20 \mathrm{~mm}^{2}\right)(\mathrm{b})$.

A detailed modeling of the RGXBPM will be possible with Monte Carlo simulation codes for photon transport, such as PENELOPE [7] and HEED [8], once these programs will have proper models for low energy polarized x-ray radiation at low gas pressures.

\section{Prototype Design}

A RGXBPM prototype was designed with dimensions and potentials which comply with requirements of uniformity of the electrical field. The CST Particle Studio ${ }^{\mathrm{TM}}$ program was used for electrostatic modeling and to define ion and electron particle trajectories [9]. The length of the RGXBPM electrodes is $80 \mathrm{~mm}$, height is $40 \mathrm{~mm}$, and distance between electrodes is $20 \mathrm{~mm}$. A guide electrode (E) was placed in the middle between repeller electrode and mesh electrode to improve field quality, Fig. 1a. The Hamamatsu F4772-01 MCP was used, which has dimensions of $55 \times 8 \mathrm{~mm}^{2}$ and a channel diameter of $12 \mu \mathrm{m}$. Two RGXBPM prototypes with two different signal readouts were built. The RGXBPM with an optical readout used an MCP with phosphor screen, the RGXBPM with current readout used an MCP with split sow-tooth electrode. For RGXBPM with an optical readout a PCO PixelFly 12 bit CCD camera, of $6.45 \mu \mathrm{m}$ pixel size, was equipped with $50 \mathrm{~mm}$ focal length objective to image the beam profile from the phosphor screen. The magnification of the optical system was chosen in order to have maximum field of view while not compromising the special resolution, which was $15 \mu \mathrm{m} / \mathrm{pixel}$.

\section{TEST RESULTS}

To test the RGXBPM performance, it was installed at the ESRF ID6 beamline on xy-stage, $29 \mathrm{~m}$ from the center of the straight section. Straight section included three planar undulators: two with magnetic period length of $42 \mathrm{~mm}$, and one with $34 \mathrm{~mm}$ period length. A $300-\mu \mathrm{m}$-thick diamond window was located at $23 \mathrm{~m}$. Slits were located at 26 $\mathrm{m}$. In order to reduce forward and back scattering, 40-mm-long copper collimators were installed before and after of the RGXBPM, with 6 and 8 -mm-diameter collimators respectively. Slit sizes were set to $2 \mathrm{~mm}$ vertically by $3 \mathrm{~mm}$ horizontally, which allow most of the undulator radiation to pass through the RGXBPM.

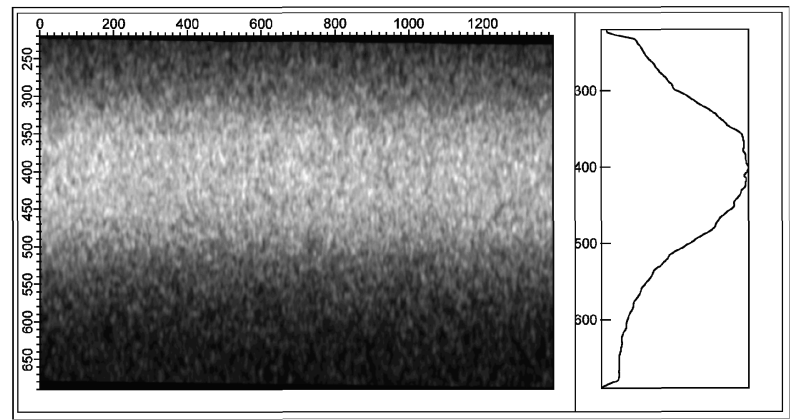

FIGURE 4. RGXBPM with optical readout. Beam profile imaged by the CCD camera from the phosphor screen, undulator u42, gap $20 \mathrm{~mm}$. 
Results of the RGXBPM test with optical readout is presented at Fig. 5a. Time of the CCD exposure was 300 $\mathrm{ms}$, for each image of the beam profile, Fig. 4, the center of gravity was calculated. The right side of Fig 5a represents a center of gravity time scan for undulator u 42 at gap of $20 \mathrm{~mm}$, the left side is for undulator u34 at gap of $20 \mathrm{~mm}$. The first fundamental harmonic and total power are $3.6 \mathrm{keV}, 7 \mathrm{keV}$ and $625 \mathrm{~W}, 227 \mathrm{~W}$ at $90 \mathrm{~mA}$ storage ring current, for u42 and u34 correspondingly. As can be seen in both cases a $5 \mu \mathrm{m}$ vertical step of the RGXBPM can be clearly resolved. At the time of measurement, the vacuum at the downstream ion pump was $2.510^{-7}$ mbar, there was no possibility to measure vacuum directly in the RGXBPM, storage ring current was $68 \mathrm{~mA}$. For ions detection, the repeller, guide and mesh electrode potentials were set to $3100 \mathrm{~V}, 1600 \mathrm{~V}$ and $-100 \mathrm{~V}$, correspondingly. The MCP input was grounded, output potential was set at $1400 \mathrm{~V}$, and phosphor screen potential was set at $3000 \mathrm{~V}$.

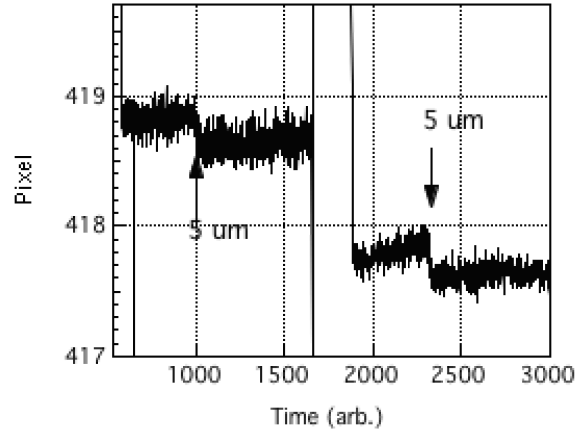

(a)

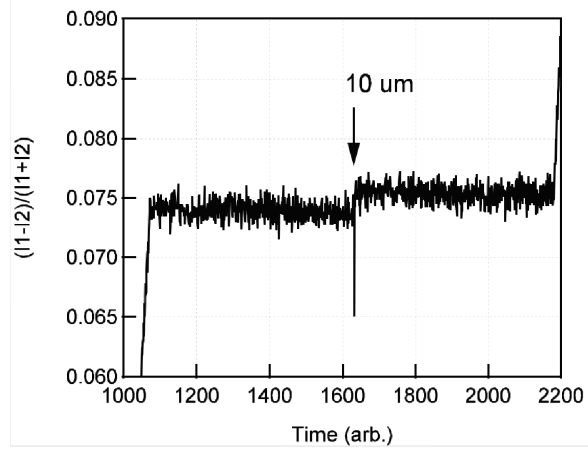

(b)

FIGURE 5. RGXBPM with optical readout (a). Center of gravity time scan, undulator u42 gap of $20 \mathrm{~mm}$ (left step) and u34, gap of $20 \mathrm{~mm}$ (right step). RGXBPM with split electrode readout (b). Normalized signal $\left(\mathrm{I}_{1}-\mathrm{I}_{2}\right) /\left(\mathrm{I}_{1}+\mathrm{I}_{2}\right)$ is shown for undulator u34, at gap of $20 \mathrm{~mm}$.

Results of the RGXBPM test with split electrode readout is presented at Fig. 5b, where normalized signal $\left(\mathrm{I}_{1}-\mathrm{I}_{2}\right) /$ $\left(\mathrm{I}_{1}+\mathrm{I}_{2}\right)$ is shown for undulator $\mathrm{u} 34$ at gap of $20 \mathrm{~mm}$. At the time of measurement, the vacuum at the downstream ion pump was $4.710^{-8} \mathrm{mbar}$, storage ring current was $80 \mathrm{~mA}$. For ions detection the repeller, guide and mesh electrode potentials were set to $4000 \mathrm{~V}, 1000 \mathrm{~V}$ and $-1900 \mathrm{~V}$, correspondingly. The MCP input potential was $-1600 \mathrm{~V}$, output potential was $-100 \mathrm{~V}$. Split electrodes were grounded through electrometers, each electrode's signal level was $\sim 2$ $\mu \mathrm{A}$. As can be seen from Fig. 5b, a $10 \mu \mathrm{m}$ vertical step of the RGXBPM is resolved.

\section{ACKNOWLEDGMENTS}

The authors would like to acknowledge J.-C. Biasci and the team of technicians for their outstanding support while RGXBPM tests were conducted at the ESRF ID6 beamline. The authors also would like to acknowledge T. Martin, C. Detlefs and H.-C. Wille for their help in organization of RGXBPM tests at the ESRF.

\section{REFERENCES}

1. O. Singh and G. Decker, "Operational Experience with X-ray Beam Position Monitors at the Advanced Photon Source", Proceedings of the PAC 2001, Chicago, USA, 2001, IEEE 0-7803-7191-7/01, pp 539-543.

2. J. Krempask $ॅ$, M. Böge, T. Schilcher, V. Schlott, T. Schmidt, "The use of Photon Monitors at the Swiss Light Source", Proceedings of EPAC 2004, Lucerne, Switzerland, 2004, pp 2520-2522.

3. F. Hornsta, W. H. Deluca, "Nondestructive beam profile System for the Zero Gradient Synchrotron", Proceedings Six International Conference on High Energy Accelerators, M. I. I., Cambridge, MA, 1967, p. 374.

4. G. I. Budker, G. I. Dimov, V. G. Dudnikov, Sov. At. Energy, 22, 441 (1967).

5. K. Wittenburg, "Experience with the Residual Gas Ionization Beam Profile Monitors at the DESY Proton Accelerators", Proceedings of EPAC 1992, Berlin, 1992, p. 1133.

6. W. H. Deluca, "Beam Detection using Residual Gas Ionization", IEEE NS, 16, 813- 822 (1969).

7. F. Salvat, J. M. Fernández-Varea, J. Sempau, "PENELOPE - A Code System for Monte Carlo Simulation of Electron and Photon Transport", Workshop Proceedings Issy-les-Moulineaux, France, 7-10 July 2003, pp. 1 - 253.

8. I.B. Smirnov, "Modeling of ionization produced by fast charged particles in gases", Nucl. Instr. and Meth. A, 554, 474-493 (2005).

9. CST Particle Studio ${ }^{\mathrm{TM}}$, CST GmbH, Bad Nauheimer Str. 19, Darmstadt, Germany. 